\title{
Cryoelectron microscopy of vitrified sections: a new challenge for the analysis of functional nuclear architecture
}

Accepted: 29 September 2005/Published online: 13 December 2005 (C) Springer-Verlag 2005

\begin{abstract}
Cryoelectron microscopy of vitrified sections has become a powerful tool for investigating the fine structural features of cellular compartments. In the present study, this approach has been applied in order to explore the ultrastructural morphology of the interphase nucleus in different mammalian cultured cells. Rat hepatoma, Chinese hamster ovary and Potorus kidney cells were cryofixed by high-pressure freezing and the cryosections were examined at low temperature by transmission electron microscopy. Our results show that while the contrast of nuclear structural domains is remarkably homogeneous in hydrated sections, some of them can be recognised due to their characteristic texture. Thus, condensed chromatin appears finely granular and the perichromatin region contains rather abundant fibro-granular elements suggesting the presence of dispersed chromatin fibres and of perichromatin fibrils and granules. The interchromatin space looks homogeneous and interchromatin granules have not been identified under these preparative conditions. In the nucleolus, the most striking feature is the granular component, while the other parts of the nucleolar body, which appear less contrasted, are difficult to resolve. The nuclear envelope is easily recognisable with its regular perinuclear space and nuclear pore complexes. Our observations are discussed in the context of results obtained by other, more conventional electron microscopic methods.
\end{abstract}

Keywords Cell nucleus · Nucleolus · Cryofixation · Vitrified sections $\cdot$ Cryoelectron microscopy

C. Bouchet-Marquis $\cdot$ J. Dubochet $\cdot$ S. Fakan $(\bowtie)$ Centre of Electron Microscopy, University of Lausanne, Bugnon 27, 1005 Lausanne, Switzerland E-mail: sfakan@unil.ch

Tel.: +41-21-6925052

Fax: + 41-21-6925055

\section{Introduction}

The functional architecture of the cell nucleus has been extensively studied for several decades using various methods of ultrastructural research. Different preparative procedures have been applied to first explore the fine structural morphology of the nucleus and further to investigate the role of nuclear structural constituents in nuclear functions by means of cytochemistry, high-resolution autoradiography, immunoelectron microscopy and molecular in situ hybridisation. These approaches made use of aldehyde-fixed cells either embedded in epoxy or acrylic resins or rapidly frozen and processed by cryoultramicrotomy, or of cryofixed samples cryosubstituted in the absence of chemical fixatives and embedded in different types of resins (review in Fakan 2004). In all these cases, the specimen was either submitted to chemical cross-linking or to at least partial dehydration, giving rise to some shrinkage of the biological material (see e.g. Hayat 2000). The development of methods allowing one to cryofix the specimen in vitreous state and observe it at a very low temperature in hydrated thin cryosections (cryoelectron microscopy of vitrified sections (CEMOVIS); review in Dubochet et al. 1988; Al-Amoudi et al. 2004) opened a new way of investigating cellular structures under conditions where the specimen is observed as close as possible in its native state. A long-lasting effort aimed at the application of this method to chromatin and nuclear structure has been made (Bednar et al. 1995; McDowall et al. 1983, 1986; Richter 1992; Sartori Blanc et al. 1998; Sartori Blanc 1997; Woodcock 1994).

In this work, we have analysed the intranuclear architecture of several types of mammalian cultured cells using CEMOVIS and compared our observations with results provided by the application of other more conventional ultrastructural approaches. 


\section{Materials and methods}

\section{Cell culture}

Chinese hamster ovary (CHO), Potorus kidney (PtK2) or rat hepatoma (HTC) cells were grown at $37^{\circ} \mathrm{C}$ with $5 \% \mathrm{CO}_{2}$ in $50-\mathrm{ml}$ Falcon tissue culture flasks. $\mathrm{CHO}$ and PtK2 cells grew in Minimum Essential Medium (MEM, Gibco, Grand Island, NY, USA) with Earl's salts, whereas HTC cells were grown in D-MEM (Gibco) both supplemented with $10 \%$ foetal calf serum (FCS), $2 \% \mathrm{~L}-$ glutamine, $50 \mu \mathrm{g}$ of penicillin and 50 units of streptomycin per millilitre.

\section{High-pressure freezing}

After $24 \mathrm{~h}$ of growth, cells at about $80 \%$ confluence were removed from the incubator and washed twice in warm $\left(37^{\circ} \mathrm{C}\right)$ phosphate buffer saline (PBS). They were mechanically detached from the surface of the flask using a cell scraper in $5 \mathrm{ml}$ PBS. This suspension was centrifuged for $5 \mathrm{~min}$ at $1,000 \mathrm{rpm}$ to form a pellet. Supernatant was removed and the cells were resuspended in $200 \mu \mathrm{l}$ of $20 \%$ Dextran/ PBS solution (Sigma, St. Louis, MO, USA, 40,210 Da). After 5 min, a homogenised suspension was sucked into a copper tube with an inner diameter of $300 \mu \mathrm{m}$ and vitrified in the LEICA EM PACT (Leica, Vienna, Austria) under a pressure of 2,000 bar by a jet of liquid nitrogen $\left(-192^{\circ} \mathrm{C}\right)$, applied on both sides of the tube at the same time (Studer et al. 2001). The vitrified cells were stored in liquid nitrogen.

\section{Cryoultramicrotomy}

Tubes containing vitrified cells obtained by highpressure freezing were mounted in an FCS cryochamber of an ultracut UCT microtome (Leica, Vienna, Austria). They were trimmed in pyramid shape in order to obtain a square surface (100 $\mathrm{nm}$ side) using a $45^{\circ}$ cryotrimming knife (Diatome, Bienne, Switzerland). Then, $50 \mathrm{~nm}$ (nominal feed) thin sections were obtained using either a $45^{\circ}$ or $35^{\circ}$ cryodiamond knife (Diatome) with a clearance angle of $6^{\circ}$. The cutting was performed at $-145^{\circ} \mathrm{C}$ with a cutting speed of $0.6 \mathrm{~mm} / \mathrm{s}$. During cutting, an anti-static device (Diatome) was used to enhance the sliding of the sections on the surface of the knife. The sections were collected on 1,000 mesh carbon-coated grids and punched between two cooled polished metal surfaces in order to attach them to the carbon film. The grids were immediately observed in the electron microscope to limit section contamination by ice crystals deposited during storage.

\section{Cryoelectron microscopy}

The grids were cryotransferred at liquid nitrogen temperature into a Gatan cryoholder (Gatan, Warrendale, PA, USA) allowing an amorphous state preservation of the specimen during imaging by stabilising the temperature at about $-180^{\circ} \mathrm{C}$. Vitrification of the specimen was tested by electron diffraction. The observations were performed on a cryotransmission electron microscope Phillips CM100 (Phillips, Eindhoven, the Netherlands) at $80 \mathrm{kV}$ in minimal beam exposure conditions $(<1,000$ electrons per $\mathrm{nm}^{2}$ per micrograph). The images were recorded on Kodak SO-163 film at various magnifications and the negatives were scanned on an Epson Expression 1680 pro film scanner with a 1,600 DPI resolution. Contrast of the images was enhanced if needed by Adobe Photoshop-CS 8.0.1. No other image modification was made. The thickness of the sections and the relative density of domains were determined from optical density measurements (Sartori Blanc et al. 1998).

\section{Quantification}

The diameter of the nucleolar granules and of the ribosomal particles was measured with Photoshop. Based on measurements of hundred particles for each type, the standard deviation was calculated online on the website for statistical computation of the University of Vassar (Vassar College, Poughkeepsie, NY, USA, http://faculty.vassar.edu/lowry/VassarStats.htm). The degree of compression of the different particles has not been taken into account in the measurements.

Fig. 1 An HTC cell nucleus in interphase observed by CEMOVIS The nucleolus is characterised by its granular component $(g)$ intermingled with less-contrasted areas, the fine structure of which, seems to be of fibrillar nature $(f)$. It is surrounded by nucleolusassociated condensed chromatin $(*)$, which links it to the nuclear envelope of a cytoplasmic invagination (in) into the nucleus. Condensed chromatin is located either at the periphery of the nucleus or within the nucleoplasm, exhibiting its characteristic fine granular structure. This domain is delineated for clarity. Fibrogranular material is visible in the periphery of the condensed chromatin, the perichromatin region (white short arrows). Interchromatin space $(I S)$ appears as a relatively homogeneous domain. The nuclear envelope (white arrows) contains nuclear pore complexes (white arrowheads). The part of the cytoplasm visible in the invagination contains several vesicles $(v s)$ and microtubules (waved arrows) oriented in different directions appearing more elliptical (double waved arrows) due to the compression of the section. Crevasses (black arrows) seen as local compression artefacts of the section, are oriented along a direction perpendicular to knife marks (large black arrow). The carbon-supporting film is broken where indicated (large white arrow) and places where it is folded $(c f)$ are visible because of their high contrast. Sometimes, a fracture of the section along the nuclear envelope (curved large arrow) generally occurring along a membrane is seen. Ice crystals (small arrows) deposited at the surface of the section are seen as dense bodies of various sizes. Scale bar represents $500 \mathrm{~nm}$ 


\section{Results}

The cellular structures in the cryosections examined in a hydrated state usually exhibit a low contrast. This is essentially due to the averaged uniform density of the crowded biological material surrounded by water.
When compared with more conventional preparative procedures, including the different types of fixation followed by a dehydration step and final contrasting of thin sections, cryoelectron microscopy precludes the use of contrasting agents applied to the sections. Therefore, hydrated cryosections exhibit contrast defined by the biological material's own density. Furthermore,

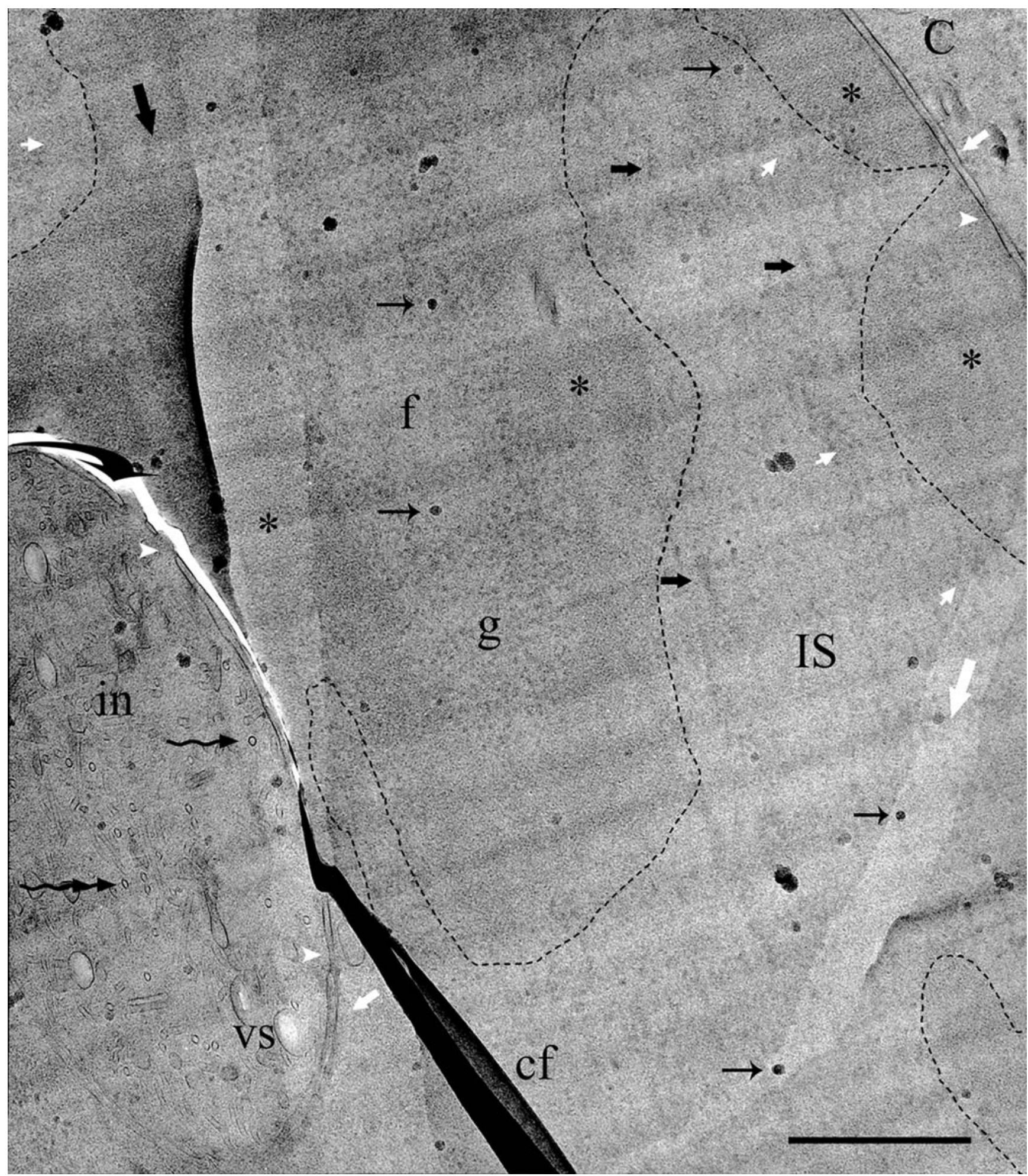


compared to plastic sections, vitreous sections are prone to more apparent cutting artefacts (Fig. 1). The parallel lines running at the surface of the section are knife marks produced by imperfections at the edge of the diamond knife. The section is compressed along the cutting direction with a proportional increase of the section thickness. Consequently, all the dimensions are reduced by the compression factor along the cutting direction, whereas they are conserved in the perpendicular direction. Circular objects such as microtubules tend to appear more elliptical in the section (Fig. 1). Crevasses are fractures oriented perpendicularly to the cutting direction (Figs. 1, 2). They can be limited by carefully chosen cutting conditions, but they are rarely completely avoided. They tend to be ironed away by the high electron dose, but this cosmetic improvement is paid by the loss of fine structures. Sometimes, sections are broken, generally along a membrane (Fig. 1). The film support can also break and fold under the section (Fig. 1). Even under strictly controlled atmosphere in the microtome room, ice crystals of various sizes can deposit on the surface of the section (Figs. 1, 2 ). They can be unambiguously identified on stereo pairs (not shown), and based on such an experiment, they can be recognised due to their characteristic shape and high contrast.

An overview of an HTC cell interphase nucleus is shown in Fig. 1. Various structural domains can be observed in the nucleus. The nucleolus is characterised by zones of large granules of $18.6 \pm 2.3 \mathrm{~nm}$ (average of 100 particles \pm SD) in size intermingled with or, more often, located around less contrasted areas, the fine structures of which, although difficult to resolve, seem to be of fibrillar nature. The low contrast of these areas does not allow one to distinguish between fibrillar centre and the dense fibrillar component, as the latter does not exhibit the high density usually observed in sections of aldehyde-fixed cells. A layer of condensed, nucleolus-associated chromatin surrounds the nucleolus and links it to the nuclear periphery.

Condensed chromatin occurs both on the nuclear periphery and within the nucleoplasm and is represented by relatively well-delimited areas of finely granular, rather homogeneous material easily identifiable compared to interchromatin space, thanks to its texture. At a higher magnification (Figs. 2, 3), condensed chromatin, although appearing less contrasted compared to sections obtained by more conventional techniques, can easily be distinguished from other nuclear structures thanks to its characteristic fine granularity. The homogeneous graininess of the condensed chromatin is best visible at higher defocusing, when dimensions in the $11 \mathrm{~nm}$ range are imaged with higher contrast. In general, there is no discernable structure of larger dimensions. While PtK2 cells (Figs. 2, 3c) mainly exhibit large condensed chromatin areas often located to the nuclear periphery, condensed chromatin regions tend to be smaller and more numerous in $\mathrm{CHO}$ and
HTC cells and occur either at the nuclear periphery or within the nucleoplasm.

The border zone of condensed chromatin, the perichromatin region, is rich in fibro-granular material. Decondensed chromatin, previously shown as being transcriptionally active (review in Fakan 2004; Spector 2003), contributes to this region, although it cannot be morphologically distinguished from ribonucleoprotein fibrils. Moreover, structural elements morphologically similar to the perichromatin fibrils found in the stained sections can be observed in the perichromatin region. They are relatively abundant (Figs. 2, 3) and their frequency gradually decreases towards the interchromatin space. In general, crosssectioned perichromatin fibrils present a smaller size than perichromatin granules (Fakan and Puvion 1980). Sometimes a fibre seems to loop out of condensed chromatin (Fig. 2b), bearing a fibril decorated by small granules of irregular shape. Furthermore, the perichromatin region contains granules with a size in the range of $30 \mathrm{~nm}$ to about $55 \mathrm{~nm}$, which likely correspond to perichromatin granules. Some of them can be identified at several locations in close contact with condensed chromatin (Fig. 2) showing an internal filamentous structure (Figs. 2, 3). Sometimes, a granule can be observed in the vicinity of a nuclear pore complex (Fig. 2).

The interchromatin space (Figs. 1, 2, 3) looks relatively homogeneous with only few clearly discernable structures. Moreover, we have not been able to clearly visualise clusters of interchromatin granules, a characteristic structural constituent occurring within the interchromatin space of all mammalian cells so far studied by means of different more conventional preparative procedures (review in Fakan 2004; Spector 2003).

The nuclear membrane is well preserved with a regular perinuclear space filled with material (Fig. 1). Nuclear pore complexes are visible on a membrane

Fig. 2 a A PtK2 cell nucleus $(N)$ presenting three large perinuclear condensed chromatin areas $(*)$. Two lipid bi-layers form the nuclear envelope showing a regular perinuclear space and a nuclear pore complex (white arrowhead). Bundles of longitudinally oriented microtubules (waved arrow), homogenously distributed ribosomes (ri) and endoplasmic reticulum $(E R)$ are visible in the cytoplasm $(C)$. Condensed chromatin border (white short arrows) appears irregular and exhibits numerous fibrils (sharp arrowheads) and granules (open arrowheads). Interchromatin space (IS) appears finely structured with low contrasted fibro-granular material where no characteristic domains like interchromatin granules are recognised. Some ice crystals (small arrows) deposit at the surface of the section during its preparation. Crevasses appear at the bottom right corner of the micrograph (arrow). Scale bar represents $200 \mathrm{~nm}$. b An enlarged view of the area delimited in (a), where a thin fibre seems to loop out of the condensed chromatin (*). A thicker fibril (arrowhead) decorated by particles is attached to it. This could correspond to a transcription complex as suggested on a schematic drawing in (b): condensed chromatin is represented in yellow, RNA polymerase complex in blue, the transcript in black, the hnRNP protein particles in red and the perichromatin region in green 
surrounding a cytoplasmic invagination (Fig. 1). The invagination contains numerous microtubules arranged in various directions as well as several vesicles. Planar membranes oriented along the viewing direction are the most conspicuous features seen in vitreous sections. This is typically the case for the nuclear envelope, which
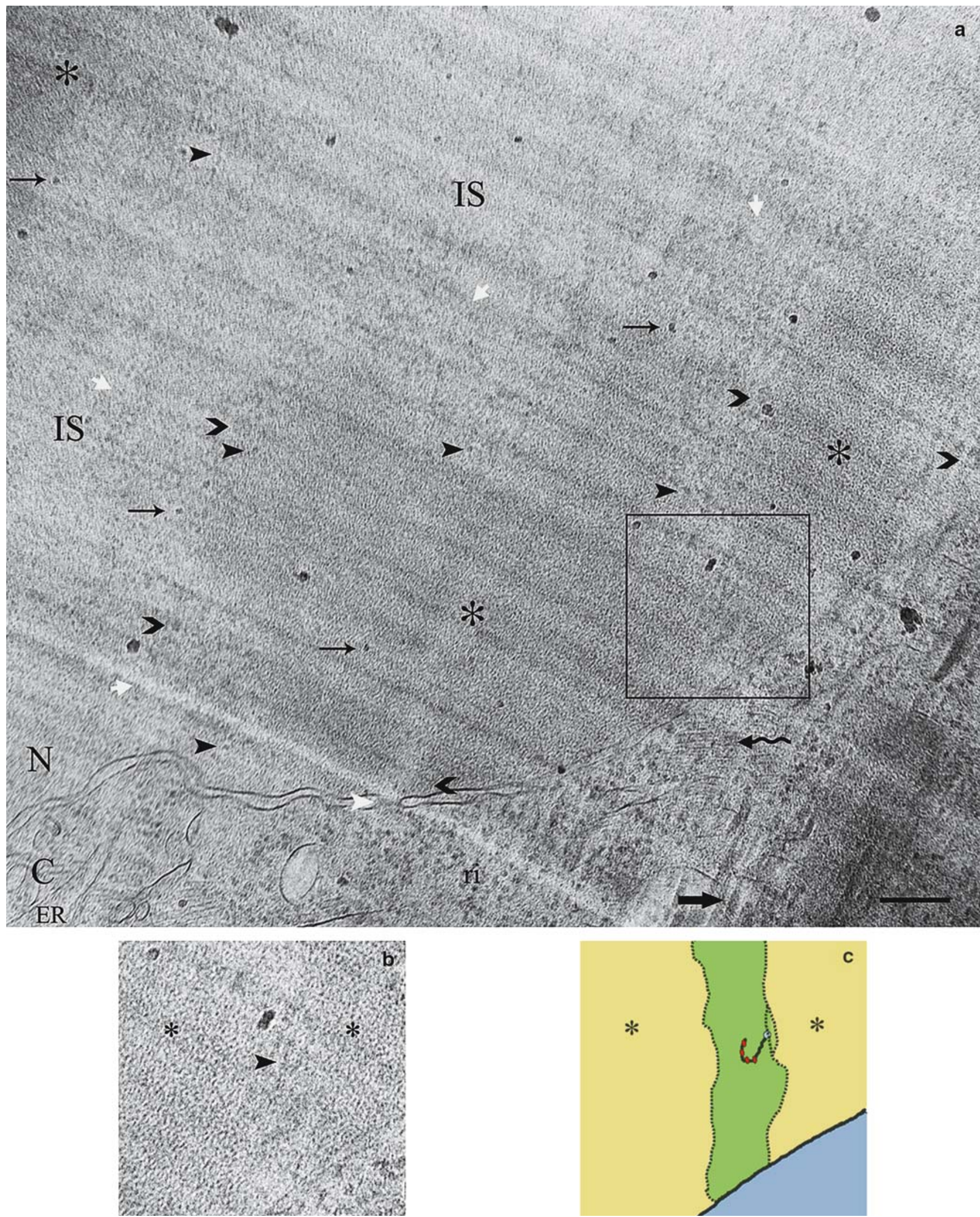


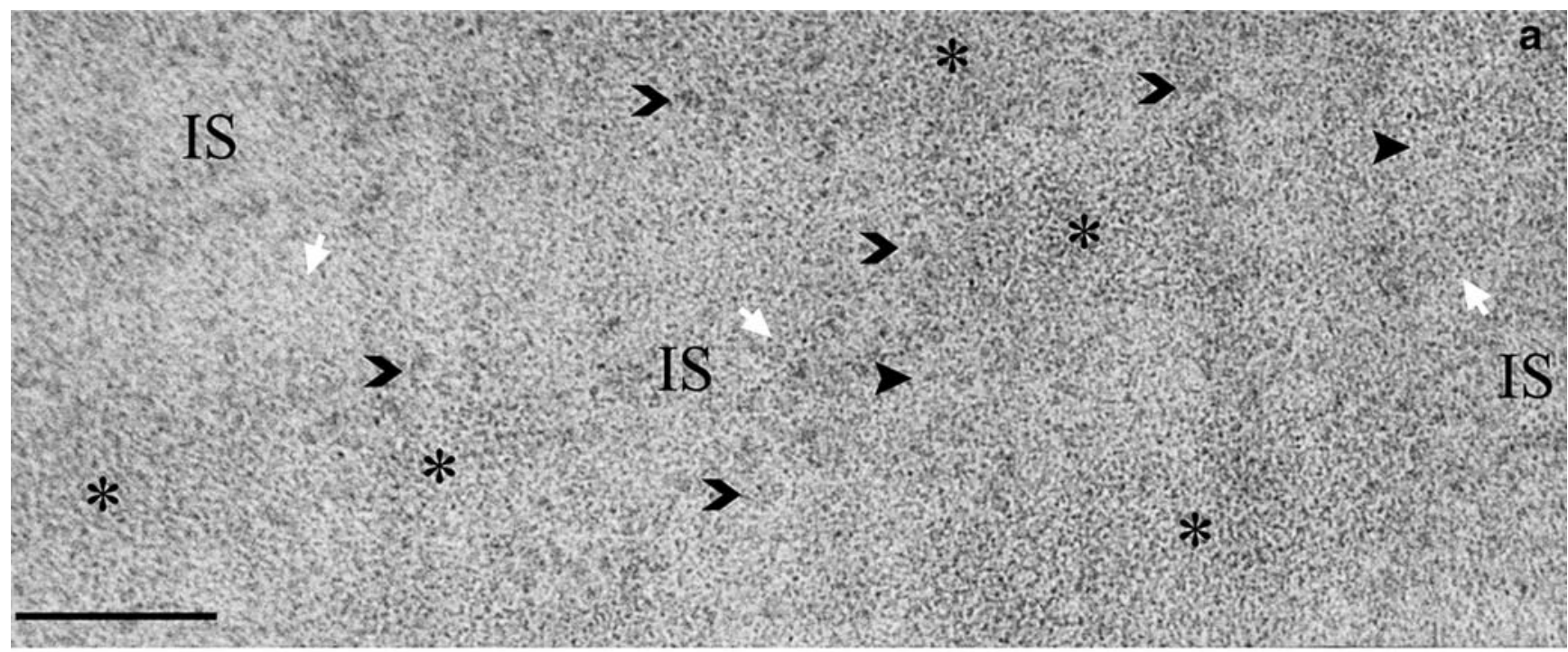

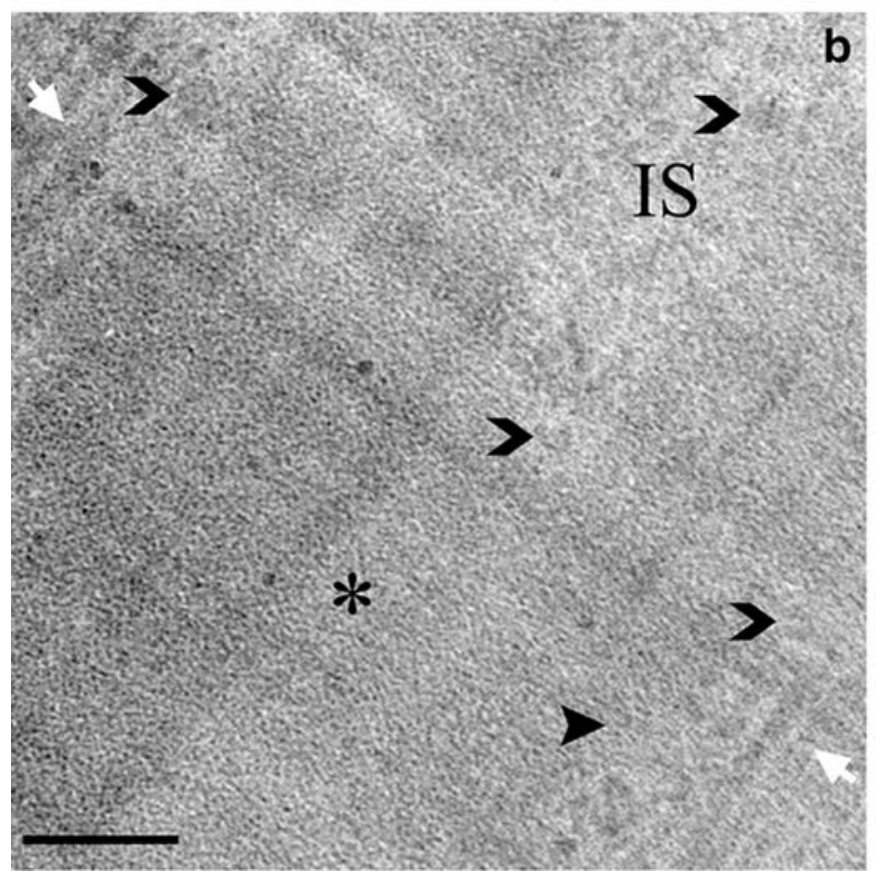

Fig. 3 A cryosection of a $\mathrm{CHO}$ cell nucleus. In a and c, condensed chromatin $(*)$, recognisable by its characteristic fine granularity, occurs as small numerous islands inside the nucleoplasm, surrounded at its periphery by a narrow region (white short arrows) of small granules and fibrils (sharp arrowheads). Many larger granules (open arrowheads) in the range of $40 \mathrm{~nm}$ (perichromatin granules)

appears, when it is not damaged by fracture or crevasses, as two $40-\mathrm{nm}$ distant parallel lines interspersed with easily identifiable, about $100-\mathrm{nm}$, large nuclear pores (Figs. 1, 2, 4). The density of the perinuclear space, between the membranes, is the same as in the neighbouring cytoplasm and nucleoplasm. Sometimes, the material occurring in this space forms a line (Fig. 1) in its central region. In general, nuclear pores are characterised by a zone of fuzzy density without membranous delineation. In most cases, there are no characteristic well-contrasted structures associated with nuclear pore complexes though low contrast granules, fibres or other features are

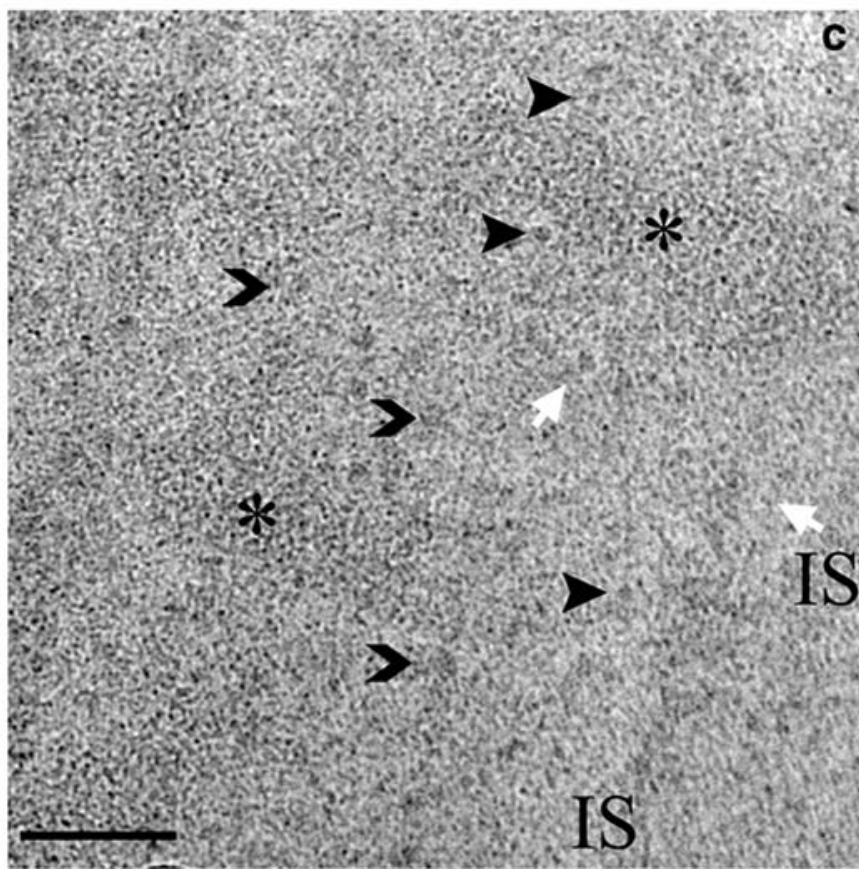

can also be identified. In PtK2 cell nucleus shown in (b), IS indicates a narrow invagination of interchromatin space into a large condensed chromatin area, where perichromatin regions from each side are close together. Perichromatin granules in close contact with condensed chromatin can appear (open arrowheads). Scale bars represent $200 \mathrm{~nm}$

visible (Fig. 4). Moreover, previously reported well-defined components of the nuclear pores (review in Pante and Aeby 1995a), such as the nuclear baskets, are not discernable.

\section{Discussion}

Our analyses of frozen hydrated ultrathin sections of nuclei in different mammalian cell types give rise to the following observations: 


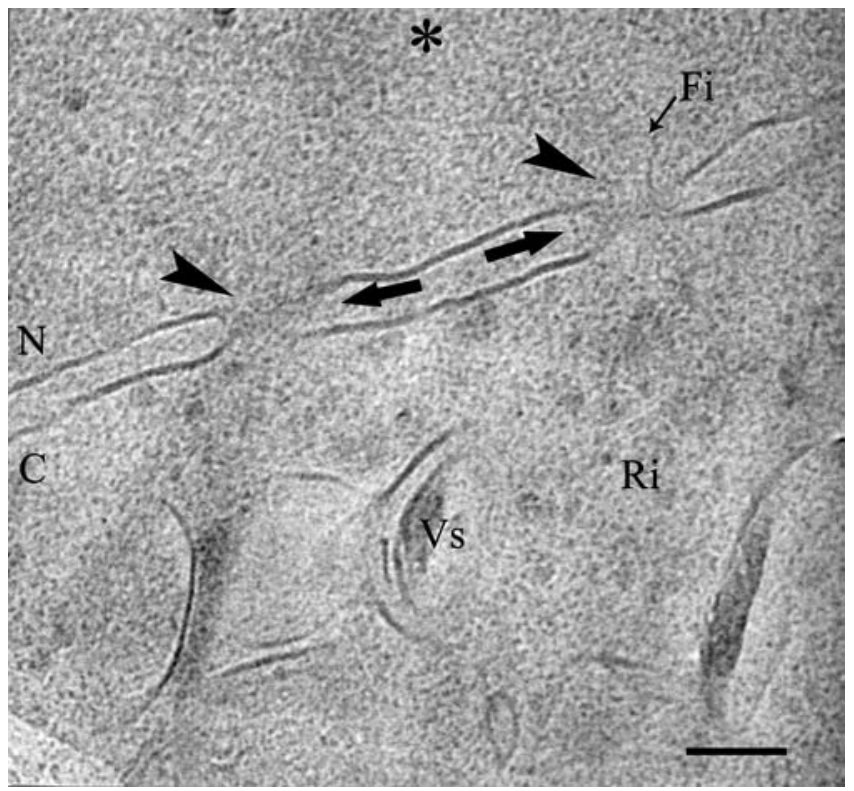

Fig. 4 A CHO cell with a nuclear envelope composed of two lipid bi-layers separated by a distance of $40 \mathrm{~nm}$ in average. Two nuclear pore complexes (sharp arrowheads) present a 100-nm-wide aperture. Vesicles $(V S)$ and ribosomes (Ri) occur in the cytoplasm $(C)$. Condensed chromatin $(*)$ is discernable at the periphery of the nucleus $(N)$ close to the nuclear pore complexes, but it does not exhibit its well-defined texture because of the defocusing conditions. Structure of the pore is defined in cryosections by a zone of fuzzy density without membranous delineation (arrow) where occasionally granules of low contrast and/or fibres of various shapes $(f i)$ can appear. Scale bar represents $100 \mathrm{~nm}$

(1) The nuclear structure of cells observed here is remarkably uniform. Even condensed chromatin has a density which does not surpass surrounding domains. Contrary to what is usual with stained embedded specimens, the various nuclear domains can only be distinguished by their fine structure or characteristic texture.

(2) Condensed chromatin occurs as a relatively homogeneous domain, revealing an essentially finely granular texture of $11 \mathrm{~nm}$ average distance.

(3) The most remarkable structural constituent of the nucleolus appears to be the granular component, while the other parts of the nucleolar body consist of relatively homogeneous material.

(4) The perichromatin region occurring on the border of condensed chromatin areas contains numerous fibrillar and granular constituents considered as dispersed chromatin, perichromatin fibrils and granules.

(5) The interchromatin space appears composed of fine fibro-granular material. However, its low contrast does not allow one to reveal clusters of interchromatin granules.

(6) The nuclear pore complexes examined so far are characterised by the presence of fuzzy material interspersing the nuclear envelope and do not seem to clearly exhibit some previously described characteristic structures, such as the nuclear basket.
The most striking feature observed when comparing more conventionally prepared specimens with hydrated cryosections is the difference in density (or apparent contrast) observed in different nuclear domains. This is especially remarkable for the nucleolar dense fibrillar component. While this structural constituent is characterised by its high density in chemically fixed cells (Goessens 1984; Hozak 1995; Raska et al. 2004), it appears less dense and more dispersed in cryofixed and cryosubstituted specimens (von Schack and Fakan 1993), and is no more recognisable in the hydrated state in our present experiments. This is also the case for the fibrillar centre, which cannot be discriminated under these conditions. This phenomenon precludes the possibility to estimate to what extent the different nucleolar components are intermingled as the granular component is the only nucleolar structural constituent clearly visible in this nuclear compartment. The granules it contains are distinct and easily recognisable. Their dimension is comparable to the dimension of ribosomes (19.0 \pm $1.9 \mathrm{~nm}$, average of 100 particles $\pm \mathrm{SD}$ ), though, they are less contrasted. Clusters of interchromatin granules presenting a remarkable nucleoplasmic morphological constituent observed so far, regardless of the preparative procedure used (Fakan and Puvion 1980; Puvion and Puvion-Dutilleul 1996; Spector 2003), have not been identified in our observations. Our results suggest that many components of the nucleus are dispersed in the native state so that they are not individually visible in the crowded environment. The difference between chemically fixed and vitreous sections could come from the fact that nuclear constituents occurring relatively close together tend to aggregate due to chemical fixation and dehydration. Such aggregation may reinforce the compaction of structures, making the domains more visible.

The perichromatin region, located on the border of condensed chromatin areas, has previously been characterised as the most important functional domain in the nucleoplasm. Indeed, preferential localisation of hnRNA transcription sites has been reported there first by high-resolution autoradiography methods (Fakan and Bernhard 1971) and later by means of brominated precursors and immunoelectron microscopy (Cmarko et al. 1999; Trentani et al. 2003). Perichromatin fibrils have been characterised in situ as structures corresponding to pre-mRNA transcripts (for review see Fakan 1994) and immunocytochemical experiments indicated the presence of most pre-mRNA processing factors associated with them (Fakan et al. 1984; Puvion et al. 1984; Spector et al. 1991; Cmarko et al. 1999). Consequently, they obviously represent primary transcripts on which major processing steps take place cotranscriptionally (for review see Fakan 2004; Spector 2003). Some of the perichromatin fibrils give rise to perichromatin granules, structural elements situated almost exclusively in the perichromatin region (Monneron and Bernhard 1969). Perichromatin granules are morphologically and cytochemically comparable with Bal- 
biani ring granules, found in Chironomus salivary gland polytene nuclei (Vazquez-Nin and Bernhard 1971), and have been demonstrated to be nuclear vectors of mRNA (Daneholt 2001). Furthermore, the perichromatin region has also been determined as the site of DNA replication (for review see Jaunin and Fakan 2002). The perichromatin region occurs on the periphery of condensed chromatin areas regardless of their size and shape and also occurs within deep and often narrow invaginations inside chromatin domains (Visser et al. 2000; for review see Cremer et al. 2004). The perichromatin region as seen in hydrated sections represents the nucleoplasmic domain with the highest frequency of fibro-granular structural components. Unfortunately, this type of preparation precludes any cytochemical or contrastenhancement treatments and conclusions made so far can only be based on direct morphological observations. This is, in particular, the case for dispersed chromatin fibres, which under present conditions cannot be clearly distinguished from ribonucleoprotein containing fibrils. The presence of hnRNP proteins on perichromatin fibrils increases their contrast and makes them more easily identifiable. Fibrils will appear cut more or less longitudinally, while those which are cross-sectioned will be detected as granules of irregular size and shape often exhibiting higher contrast. Occasionally, a fibre with an attached short lateral fibril seems to loop out of the condensed chromatin (Fig. 2b, c), reminding a transcription complex visualised after spreading of active chromatin by the Miller procedure (Beyer and Osheim 1991; Fakan and Hughes 1989). However, in the absence of further evidence, this interpretation remains speculative. As mentioned above, perichromatin granules represent another remarkable structural constituent present in the perichromatin region. Our observations show such granules exhibiting a relatively variable size, like in sections of aldehyde-fixed material prepared by cryoultramicrotomy (Puvion and Bernhard 1975; Fakan et al. 1976). Moreover, they sometimes appear as consisting of small granular elements (Figs. 2, 3), reminding thus of a substructure previously reported after ESI of phosphorus (Vazquez-Nin et al. 1996). The interchromatin region examined on vitrified specimens exhibits a lower frequency of fibro-granular material than the perichromatin region (Fig. 2).

Nuclear pore complexes are known to be the gate for molecules, which transit between the cytoplasm and the nucleus. Their structure was investigated by several approaches, such as the immunocytochemical experiments (Gerace et al. 1978) on resin-embedded specimens and cryoelectron tomography on vitreous isolated specimens (Beck et al. 2004; Stoffler et al. 2003). These studies demonstrated that they are composed of a luminal spoke complex situated between a nuclear and a cytoplasmic ring, on which, filaments are attached interacting with cargos, thus facilitating their approach and transit through the complex (Rout et al. 2003). Even if the characteristic features of the nuclear pore complexes, such as the nuclear basket, are not discernable in cryohydrated ultrathin sections, the observed nuclear pore complexes appear as 100-nm-wide aperture in the nuclear envelope in agreement with earlier studies (Stoffler et al. 2003). The difference observed between a less-defined structure of a nuclear pore complex visualised in vitreous sections and the ordered arrangement inferred from more conventional preparations (Pante and Aebi 1995b) or in vitreous total preparations of isolated material may be explained by the crowded environment of the nuclear pore, which hides its specific structure in its native state, and by the fact that the native pore could be more polymorphic and mobile than suggested by conventionally prepared samples.

In order to further approach the in situ structural arrangement of domains such as the fibrillar portion of the nucleolus, the perichromatin region, the interchromatin space or the nuclear pore complex, the tomography of vitreous sections (TOVIS) probably represents the most powerful tool at the present moment. It combines the advantage of vitreous section with the possibility of high-resolution reconstruction into a 3$\mathrm{D}$ model of the section (Beck et al. 2004). A resolution better than $6 \mathrm{~nm}$ can presently be obtained on thin vitreous layers of cells. Reaching $3 \mathrm{~nm}$ with TOVIS seems to be a realistic hope for the near future. The TOVIS approach should contribute to overcome the limits of 2$\mathrm{D}$ images and make it possible to carry out reconstructed models of structural domains reflecting at high resolution, the cellular structural constituents in situ under the best preparative conditions.

Acknowledgements We would like to thank Mrs. J. Fakan and F. Voinesco for the careful preparation of cell cultures. We thank Prof. M. Trendelenburg (Heidelberg) for kindly providing PtK2 cells. This work was supported by the Swiss National Science Foundation (grant 31-64977.01 to SF) and the European 3D-EM network.

\section{References}

Al-Amoudi A, Chang JJ, Leforestier A, McDowall A, Salamin LM, Norlen LP, Richter K, Blanc NS, Studer D, Dubochet J (2004) Cryo-electron microscopy of vitreous sections. EMBO J 23:3583-3588

Beck M, Forster F, Ecke M, Plitzko JM, Melchior F, Gerisch G, Baumeister W, Medalia O (2004) Nuclear pore complex structure and dynamics revealed by cryoelectron tomography. Science 306:1387-1390

Bednar J, Horowitz RA, Dubochet J, Woodcock C (1995) Chromatin conformation and salt-induced compaction: threedimensional structural information from cryo-electron microscopy. J Cell Biol 131:1365-1376

Beyer AL, Osheim YN (1991) Visualization of RNA transcription and processing. Semin Cell Biol 2:131-140

Cmarko D, Verschure P, Martin TE, Dahmus ME, Krause S, Fu X-D, van Driel R, Fakan S (1999) Ultrastructural analysis of transcription and splicing in the cell nucleus after Bromo-UTP microinjection. Mol Biol Cell 10:211-223

Cremer T, Kupper K, Dietzel S, Fakan S (2004) Higher order chromatin architecture in the cell nucleus: on the way from structure to function. Biol Cell 96:555-567 
Daneholt B (2001) Packing and delivery of a genetic message. Chromosoma 110:173-185

Dubochet J, Adrian M, Chang J-J, Homo J-C, Lepault J, McDowall AW, Schultz P (1988) Cryo-electron microscopy of vitrified specimens. Q Rev Biophys 21:129-228

Fakan S (1994) Perichromatin fibrils are in situ forms of nascent transcripts. Trends Cell Biol 4:86-90

Fakan S (2004) The functional architecture of the nucleus as analysed by ultrastructural cytochemistry. Histochem Cell Biol 122:83-93

Fakan S, Bernhard W (1971) Localisation of rapidly and slowly labelled nuclear RNA as visualized by high resolution autoradiography. Exp Cell Res 67:129-141

Fakan S, Hughes ME (1989) Fine structural ribonucleoprotein components of the cell nucleus visualized after spreading and high resolution autoradiography. Chromosoma 98:242-249

Fakan S, Puvion E (1980) The ultrastructural visualization of nucleolar and extranucleolar RNA synthesis and distribution. Int Rev Cytol 65:255-299

Fakan S, Puvion E, Sphor G (1976) Localization and characterization of newly synthesized nuclear RNA in isolated rat hepatocytes. Exp Cell Res 99:155-164

Fakan S, Leser G, Martin TE (1984) Ultrastructural distribution of nuclear ribonucleoproteins as visualized by immunocytochemistry on thin sections. J Cell Biol 98:358-363

Gerace L, Blum A, Blobel G (1978) Immunocytochemical localization of the major polypeptides of the nuclear pore complexlamina fraction. Interphase and mitotic distribution. J Cell Biol 79:546-566

Goessens G (1984) Nucleolar structure. Int Revcytol 87:107-158

Hayat MA (2000) Principles and techniques of electron microscopy biological applications, 4th edn. Cambridge University Press, Cambridge

Hozak P (1995) Catching RNA polymerase I in flagranti: ribosomal genes are transcribed in the dense fibrillar component of the nucleolus. Exp Cell Res 216:285-289

Jaunin F, Fakan S (2002) DNA replication and nuclear architecture. J Cell Biochem 85:1-9

McDowall AW, Chang J-J, Freeman R, Lepault J, Walter CA, Dubochet J (1983) Electron microscopy of frozen hydrated sections of vitreous ice and vitrified biological samples. J Microsc 131:1-9

McDowall AW, Smith JM, Dubochet J (1986) Cryo-electron microscopy of vitrified chromosomes in situ. EMBO J 5:13951402

Monneron A, Bernhard W (1969) Fine structural organization of the interphase nucleus in some mammalian cells. J Ultrastruct Res 27:266-288

Pante N, Aebi U (1995a) Exploring nuclear pore complex structure and function in molecular detail. J Cell Sci Suppl 19:1-11

Pante N, Aebi U (1995b) Toward a molecular understanding of the structure and function of the nuclear pore complex. Int Rev Cytol 162B:225-255

Puvion E, Bernhard W (1975) Ribonucleoprotein components in liver cell nuclei as visualized by cryoultramicrotomy. J Cell Biol 67:200-214
Puvion E, Puvion-Dutilleul F (1996) Ultrastructure of the nucleus in relation to transcription and splicing: roles of perichromatin fibrils and interchromatin granules. Exp Cell Res 229:217-225

Puvion E, Viron A, Assens C, Leduc EH, Jeanteur P (1984) Immunocytochemical identification of nuclear structures containing snRNPs in isolated rat liver cells. J Ultrastruct Res $87: 180-189$

Raska I, Koberna K, Malinsky J, Fidlerova H, Masata M (2004) The nucleolus and transcription of ribosomal genes. Biol Cell 96:579-594

Richter K (1992) Cryomicroscopie électronique des sections minces vitrifiées. PhD Thesis, Université de Lausanne

Rout MP, Aitchison JD, Magnasco MO, Chait BT (2003) Virtual gating and nuclear transport: the hole picture. Trends Cell Biol 13:622-628

Sartori Blanc N (1997) Cryo-microscopie électronique de sections vitrifiées et étude de la structure du noyau cellulaire par cette technique. PhD Thesis, Université de Lausanne

Sartori Blanc N, Studer D, Ruhl K, Dubochet J (1998) Electron beam-induced changes in vitreous sections of biological samples. J Microsc 192:194-201

von Schack ML, Fakan S (1993) The study of the cell nucleus using cryofixation and cryosubstitution. Micron 24:507-519

Spector DL (2003) The dynamics of chromosome organization and gene regulation. Annu Rev Biochem 72:573-608

Spector DL, Fu XD, Maniatis T (1991) Associations between distinct pre-mRNA splicing components and the cell nucleus. EMBO J 10:3467-3481

Stoffler D, Feja B, Fahrenkrog B, Walz J, Typke D, Aebi U (2003) Cryo-electron tomography provides novel insights into nuclear pore architecture: implications for nucleocytoplasmic transport. J Mol Biol 328:119-130

Studer D, Graber W, Al-Amoudi A, Eggli P (2001) A new approach for cryofixation by high-pressure freezing. J Microsc 203:285-294

Trentani A, Testillano PS, Risueno MC, Biggiogera M (2003) Visualization of transcription sites at the electron microscope. Eur J Histochem 47:195-200

Vazquez-Nin G, Bernhard W (1971) Comparative ultrastructural study of perichromatin- and Balbiai ring granules. J Ultrastruct Res 36:842-860

Vazquez-Nin GH, Abolhassani-Dadras S, Echeverria OM, Boutinard Rouelle-Rossier V, Fakan S (1996) Phosphorus distribution in perichromatin granules and in surrounding nucleoplasm as visualized by electron spectroscopic imaging. Biol Cell 87:171-177

Visser AE, Jaunin F, Fakan S, Aten JA (2000) High resolution analysis of interphase chromosome domains. J Cell Sci 113:2585-2593

Woodcock CL (1994) Chromatin fibers observed in situ in frozen hydrated sections. Native fiber diameter is not correlated with nucleosome repeat length. J Cell Biol 125:11-19 